PREPRINT - PLEASE DO NOT CITE WITHOUT PERMISSION

Emerging Self-Representation Presents a Challenge for Perspective Tracking in Infancy

Emanuela Yeung, Dimitrios Askitis, Velisar Manea, and Victoria Southgate

Department of Psychology, University of Copenhagen

Correspondence regarding this paper can be addressed to Emanueula Yeung, Department of Psychology, University of Copenhagen, Øster Farimagsgade 2A, 1353 Copenhagen K, Denmark. Email: eyeung@psy.ku.dk 


\begin{abstract}
The capacity to track another's perspective is present from early in life, with young infants ostensibly able to predict others' behaviour even when the self and other perspective are at odds. Yet, infants' abilities are difficult to reconcile with the well-documented challenge that older children face when they need to ignore their own perspective. Here we provide evidence that it is the emergence of self-representation, from around 18 months, that likely creates a perspective conflict between self and other. Using mirror self-recognition as a measure of self-awareness and pupil dilation to index conflict processing, our results show that mirror recognisers perceive greater conflict when viewing a scenario in which the self and other have divergent perspectives, specifically when the conflict between self and other is salient. These results suggest that infants' perspective tracking abilities may benefit from an initial absence of self-representation.
\end{abstract}




\section{Emerging Self-Representation Presents a Challenge for Perspective Tracking in Infancy}

Young infants are ostensibly able to predict others' behaviour even when the self and other perspectives diverge (Baillargeon et al., 2010; Choi et al., 2018; Luo \& Johnson, 2009), a feat that is difficult to reconcile with the well-known difficulty that 3-year-olds face when they need to ignore their own perspective (Birch \& Bloom, 2007). A particular challenge for explaining infants' abilities is to account for the apparent absence of maturational factors like inhibitory control, which play a role in managing perspective conflict in older children (Devine \& Hughes, 2014).

The false belief task is the paradigmatic case of perspective conflict because, to make the correct inference, one cannot rely on one's own representation and many studies have demonstrated some involvement of inhibitory resources in success on verbal mentalizing tasks (VMTS; Devine \& Hughes, 2014). This relationship likely stems from the need to deal with two conflicting representations: one derived from one's own knowledge of reality and the other from encoding the other's perspective. To resolve this conflict, various accounts appeal to conflict monitoring and management mechanisms (Leslie et al., 2004). Neuroimaging work supports this view by demonstrating that brain regions implicated in inhibition are required for success on perspective conflict tasks (Hartwright et al., 2015; Samson et al., 2005) and recruited at a point where the participant needs to make a judgement (McCleery et al., 2011). Given that young infant's inhibitory skills are likely to be less developed than those of pre-schoolers (Hendry et al., 2016; Müller \& Kerns, 2015), it remains difficult to explain how infants as young as 6 months of age can make accurate predictions in scenarios containing perspective conflict (Luo \& Baillargeon, 2007; Southgate \& Vernetti, 2014).

One explanation could be that the non-verbal measures with which infants' abilities tend to be measured do not demand inhibition of the self perspective (Baillargeon et al., 2010). While this would be a neat solution, it seems unlikely that the nonverbal nature of the task circumvents the need for self-perspective inhibition since dual-task manipulations, targeting executive functions, impair adults' performance on an analogous nonverbal perspective conflict task (Schneider et al., 
2012). Furthermore, manipulating the presence or absence of the object, designed to target inhibitory demand, impairs both children and adults' ability to make belief-based action predictions on nonverbal task (Wang \& Leslie, 2016).

Recently, an alternative account has argued that infants' precocious success on tasks involving perspective conflict may be explained by an altercentric bias in infant cognition (Southgate, 2020). According to this hypothesis, infants can track others' perspectives because they lack a competing self-perspective. In the absence of a self-perspective, young infants may not experience the perspective conflict inherent in a false belief task. This hypothesis draws on evidence for a late emergence of cognitive self-awareness between 18 and 24 months of age and proposes that selfawareness is a prerequisite for generating a self-perspective. It further proposes that only when the child is able to generate a representation of their own perspective (e.g., the ball is in the left-hand box) does this representation become a competitor to the representation of the object cued by another agent (the ball is in the right-hand box). Thus, in the absence of the self-perspective, young infants can track the perspective of another individual without the need to inhibit or control a conflicting self-perspective (Southgate, 2020).

In the present preregistered study, we tested this hypothesis. Based on the assumption that conflict in a false-belief scenario arises because of a mismatch between the self- and otherperspective, we investigated whether experienced conflict was greater in infants who have achieved self-awareness, a presumed prerequisite for representation of the self-perspective. Our main question was whether a manipulation aimed at increasing or decreasing the demands on participants' inhibitory resources in a perspective-conflict scenario, would a) be detected only by infants who exhibit evidence of self-awareness and b) modulate action prediction only in infants who exhibit evidence of self-awareness.

In line with previous work (Hartwright et al., 2015; Samson et al., 2005; Wang \& Leslie, 2016), we manipulated inhibitory demand by either transferring an object from one box to another (high-conflict, HDfb) or removing the object from the scene altogether (low-conflict, LDfb). In both 
cases, there is a conflict in perspectives between the self and other, but that conflict is greater when the object remains in the scene as a salient reminder of the participant's own knowledge. We used the mirror self-recognition (MSR) test as an index of self-awareness, categorizing infants as either recognisers or non-recognisers. While MSR is a direct measure of physical self-recognition, there is considerable evidence that it also indexes 'objective' self-awareness, relating to self-other comparison (Kampis et al., 2021), dyadic imitation (Asendorpf \& Baudonnière, 1993), personal pronoun use (Lewis \& Ramsay, 2004), and brain indices of self-related processing (Bulgarelli et al., 2019).

We preregistered the prediction that recognisers but not non-recognisers, would perceive greater conflict on high- than low-conflict trials. To measure this, we used pupil size as an index of perceived conflict (Rondeel et al., 2015; Sirois \& Brisson, 2014), predicting greater pupil dilation in the high than low perspective conflict events in infants who evidence mirror self-recognition. Change in pupil diameter has been used as a marker of cognitive effort in both adults and infants (Hess \& Polt, 1960; Kahneman \& Beatty, 1966; Kaldy \& Blaser, 2020).

Secondly, we asked whether self-awareness would modulate belief-based action prediction, reasoning that experiencing conflict between perspectives would impair children's action prediction capabilities in the absence of mature inhibitory resources. Thus, we preregistered the prediction that infants who had achieved MSR would show a greater tendency towards incorrect action anticipation than those who had not. We further aimed to test the prediction that there would be a relationship between inhibitory control and anticipatory looking in the high-conflict condition, but only in those infants who had achieved self-representation. In other words, we reasoned that for those infants who did experience the conflict in perspectives, better inhibitory control ability would allow them to prioritise the other's perspective and make the correct anticipation.

\section{Method}


This study was preregistered on the Open Science Framework and the description of the testing protocol and planned analyses can be found here. All experimental and data processing scripts and stimuli videos can be found here.

\section{Participants}

To reach our pre-registered sample of 50 infants $(n=25$ in each group of mirror recognisers and mirror non-recognisers) we tested 80 infants in total. Participants were included based on providing sufficient eye-tracking data for analysis (described below) and completing the MSR task. Of the 50 infants included in the non-verbal mentalizing task ( $M$ age $=18.17$ months, $S D=9.83$ days, 20 females), $n=40$ also successfully completed the inhibition task.

The study was approved by the Research Ethics Committee of [blinded for review] and parents provided informed consent prior to participation.

\section{Procedure}

Infants were most often accompanied by one parent, who was asked to maintain a neutral presence throughout the session. The task order for all participants was the same: (1) the NonVerbal Perspective-Tracking Task (NVMT), (2) the Early Childhood Inhibitory Touchscreen Task (ECITT), and (3) the MSR.

\section{Non-Verbal Perspective-tracking Task}

\section{Apparatus}

Gaze and pupil diameter data were recorded from both eyes at $500 \mathrm{~Hz}$ using an Eyelink1000 Plus eye tracker (SR Research, Ontario, Canada). The stimuli were presented on a 17" monitor. Stimuli

Animations were created using Blender (Community, 2018) and Adobe Premier Pro. We ensured that our stimuli were isoluminant across the duration of each trial and between conditions, to minimize pupil changes caused by variations in luminosity. 
Infants were seated in their parent's lap, approximately $60 \mathrm{~cm}$ from the screen and Eyelink's 5-point calibration was used for each infant. Stimuli were presented using MATLAB for Mac (R2016b) and Psychtoolbox (3.0.16 Beta, SVN revision 10420).

The sequence of events is shown in Figure 1. Infants were first presented with two familiarization trials. On each trial, an agent observed a ball jumping into one of two boxes. Next, two lights illuminated together with a sound cue, and the agent reached into the box to retrieve the ball. Infants saw the agent reaching once into the left box and once into the right box with order counterbalanced across infants.

Following familiarization, test trials were presented in blocks, each consisting of 11 trials. On each test trial, the agent witnessed a ball jump into one of two boxes. A curtain was then drawn shut, occluding the agent, and the ball then jumped into the other box (false belief - high demand condition, HDfb, movie 1) or out of the scene (false belief - low demand condition, LDfb, movie 2). The curtains reopened, revealing the agent, the boxes reopened, and the lights on each box illuminated together with a sound cue. In each block, participants saw 5 videos for each false belief condition, including 2 trials where the agent reached into the box where they last saw the ball (the 'outcome') after the anticipation phase. In addition, infants saw 1 'true belief' trial in each block in which the agent witnessed the ball jumping into the second box and then reached into the box where the ball was located. The purpose of the outcome and true belief trials was to motivate infants to make anticipatory looks throughout the block (see below), but the gaze and pupil data from these trials were not used for analysis.

Individual trials were excluded if infants looked away during portions of the familiarization and/or test trials that were critical for understanding the structure of the task (see supplementary materials (SM) for further details).

\section{Pupil Diameter}

The eye-tracking data was extracted from the eye-tracker using the Eyelink IDE and custom MATLAB scripts. The raw pupil data was first converted from arbitrary units to millimeters. Next, we 
examined the data obtained from each eye for each participant and excluded data from one eye when the ratio of the pupil standard deviations between eyes was less than 1.5. The subsequent steps of our pre-processing pipeline followed the procedure described by Kret and Sjak-Shie (2019); for details, see SM. Due to preprocessing, we removed $20.7 \%$ (SD $=15.4 \%)$ of pupil data on average for each participant. We also removed trials with more than $30 \%$ of data missing, which resulted in acceptance of 835 out of 1086 trials. Our final sample consisted of infants who provided at least 3 trials per condition after pre-processing, resulting in 707 trials included in total.

Our primary dependent measure was change in pupil dilation. We calculated the change in pupil dilation by subtracting the pupil size at each timepoint from a baseline period. We defined the baseline as the average pupil diameter between $2000-5000 \mathrm{~ms}$, which corresponds to the movement of the ball from the centre of the screen into the first box and is identical in both high- and lowconflict conditions. In order to ensure that pupil data was not contaminated by noise in the baseline, we restricted the analysis of pupil data to trials that had no missing values in their baseline. This resulted in a further exclusion of 51 trials, leaving 656 trials in total.

The test phase was the period between 6.5 to $17.5 \mathrm{~s}$ of each trial. Previous studies have found that infants' pupils react more slowly than those of adults', and require more time to adapt to changes in stimuli (Pätzold \& Liszkowski, 2019; Verschoor et al., 2015). As such, the time windows for analyses of change in pupil size were adjusted to $500 \mathrm{~ms}$ after event boundaries. Because we did not have a priori information about when infants might experience a conflict between the self- and other-perspective, we pre-registered three possible windows for analysis: 1) $6.5-11.5 \mathrm{~s}$, when the ball moved from the first to the second box, 2) $11.5-14.5 \mathrm{~s}$, when the agent returned and the boxes reopened, and 3) 14.5-17.5s, when the sound and light cued for action prediction, during which the agent sat stationary behind the boxes (see Figure 1 for an overview of our task design). 
Fixations were computed online by the Eyelink system using the cognitive configuration (see Eyelink manual 2017, 4.3.9), and fixations from both eyes were used when available to assess fixation on areas of interest (AOIs) or on the screen.

We preregistered two dependent measures to analyse our anticipatory looking data. Our first dependent measure, 'first fixations', was binary and expresses whether the first box AOI that the participant fixated on within the time window of interest was the correct side. We defined an AOI that included the two boxes (see SM). The time window of interest was initially defined as the $3000 \mathrm{~ms}$ interval starting from the sound and light cue onset. However, upon reviewing the gaze data and video recordings from our participants, we found that many infants already made anticipatory saccades as the boxes reopened, $835 \mathrm{~ms}$ prior to the light cues on the boxes. Thus, we adjusted our time window of interest to begin when the boxes opened. First fixation scores were averaged across all trials per condition for each participant.

The second dependent variable was a differential looking score (DLS) calculated for each participant as cumulative fixation time to correct box $\mathrm{AOI}$ divided by the sum of cumulative fixation times to the two box AOIs. The cumulative fixation times were calculated within $3000 \mathrm{~ms}$ from the box opening cue onset. DLS scores were averaged across all trials per condition for each participant. In addition to the exclusion criteria described above, we also excluded trials where the child did not make fixations in either box $\mathrm{AOI}$ within the $3000 \mathrm{~ms}$ from cue onset.

\section{Early Childhood Inhibition Touch Screen Task}

The Early Childhood Inhibitory Touchscreen Task (ECITT; (Holmboe et al., 2021) is a computerized touchscreen task designed to assess inhibitory control in infants and toddlers (see SM for additional details regarding the materials and procedure).

\section{Mirror Self-Recognition Task}

\section{Procedure}

We conducted a MSR task (following the procedure of Bulgarelli et al., 2019; see also (Amsterdam, 1972) to assess children's ability to recognize themselves in the mirror. The testing 
procedure included four phases: 1) children were first exposed to and familiarized with the mirror prior to application of the mark, 2) the mark was applied to the child's nose surreptitiously after mirror was occluded, 3) the child was exposed to the mirror again after the mark was on their nose, and 4) the experimenter pointed to the child's reflection in the mirror and asked "Who is that?".

\section{Coding}

Children's reactions in the mirror were coded by a trained research assistant with respect to whether they touched the mark on their nose during phase 3 (mark-directed behaviour) and whether they verbalized any self-reference when seeing themselves in the mirror during phase 3 or upon request during phase 4 . Children were considered to have passed the mirror test (mirror recognisers) if they showed mark-directed behaviour, used a first-person pronoun, or used their own name. Children who did not exhibit these behaviours were considered mirror non-recognisers. None of the children touched the mark during the second phase before seeing themselves in the mirror. $50 \%$ of participants were double-coded by a second independent coder. In case of disagreement, a third coder was involved, and the final score was decided by the majority.

\section{Results}

\section{Pupil dilation}

After pre-processing, infants in our sample watched a mean of $5.46 \pm 2.05 \mathrm{HDfb}$ and $5.44 \pm$ $1.82 \mathrm{LDfb}$ trials. There was no significant difference in the number of trials included per condition $(t(49)=0.10, p=0.92)$. When split by MSR status, there were also no differences in number of trials included in both the $\mathrm{HDfb}\left(M_{\text {recognisers }}=5.00 \pm 1.98, M_{\text {non-recognisers }}=5.92 \pm 2.06, t(48)=1.61, p=0.11\right)$, and LDfb conditions $\left(M_{\text {recognisers }}=5.04 \pm 1.65, M_{\text {non-recognisers }}=5.84 \pm 1.93, t(48)=1.58, p=.12\right)$.

Figure 2 shows the mean baseline-corrected pupil diameter for the duration of the trial for both groups of participants across the two conditions. To analyse differences in pupil dilation, we pre-registered analyses using both ANOVA and functional data analysis (FDA). One participant (a 
mirror recogniser) did not have sufficient data in window 3 and was excluded from the ANOVA for this time window and the FDA analysis.

First, we looked at differences in dilation using 2 (condition: HDfb vs. LDfb) $\times 2$ (MSR status: recogniser vs. non-recogniser) repeated-measures ANOVAs, with pupil dilation averaged within each of the 3 pre-registered time windows. However, there were no significant main effects or interactions in any of the time windows (see SM for details). Although averaging across time windows or trials is common practice for other measures of gaze data, this approach may not be optimal for pupil data due to loss of information (Jackson \& Sirois, 2009). For this reason, we also pre-registered FDA (Ramsay \& Silverman, 2002) to assess whether the change in pupil dilation over time differed between our two groups and to determine the specific time windows in which differences could be observed.

The data was fitted using B-spline functions of order 4 with 58 bases. We performed a functional two by two mixed ANOVA (Ramsay \& Silverman, 2002) with MSR status as the between groups factor and demand condition (HDfb and LDfb) as the repeated measure. To determine statistical significance thresholds, we performed permutation analysis at 0.05 significance level and 10000 permutations, where the MSR and demand conditions were randomly shuffled. For each permutation, the computed F-statistics were used to form null distributions, against which our original values were compared. Between mirror groups we detected a significant difference in pupil dilation in the anticipation window between $15918-16384 \mathrm{~ms}$ and $16544-16904 \mathrm{~ms}$, indicating that mirror recognisers showed greater pupil dilation compared to mirror non-recognisers during these periods (see Figure 3a).

When we compared dilation between HDfb and LDfb conditions, there was a statistical difference in the time period where they differ visually (i.e., when the ball jumps out of the scene or into the second box). We did not find any other statistical difference in pupil dilation between the two conditions, nor in their interaction with the MSR factor (see Figures 3b-3c). However, when examining the HDfb condition separately, a functional t-test showed that baseline-corrected pupil 
dilation differed between MSR groups in the anticipation phase, specifically between 15790-16990 ms. This indicates that mirror recognisers showed greater pupil dilation than mirror non-recognisers during the anticipation window of the HDfb condition. No differences in pupil dilation between mirror status groups were detected in the LDfb condition (see Figures 4a-4b).

\section{Exploratory Analyses}

Growth Curve Analysis. To provide further support for the effect of MSR status on pupil dilation detected in the third pre-registered time window (14.5s-17.5s), we performed a post hoc growth curve analysis (GCA) to examine how the pupil reactions deviated over time. GCA is a multilevel regression model that fits orthogonal polynomials to time series data to model variations in the curve shape (Mirman, 2017). It has been increasingly used in modelling pupil data time series (Kuchinsky et al., 2013; McGarrigle et al., 2017; Wagner et al., 2019; Winn, 2016) and is ideal for investigating the temporal dynamics of pupil data that can be obscured in a time window averaging approach.

We used normalised orthogonal polynomial terms up to degree 4 to model the pupil data from $14.5 \mathrm{~s}$ to $17.5 \mathrm{~s}$ (see SM for further details). Given that our FDA analysis revealed effects only in the HDfb condition, we restricted our analysis in this condition. We considered the full model with mirror status as a main effect and all its interactions with polynomial terms (see Table 1). Based on a Likelihood Ratio Test, the full model fit the data better than the null model (i.e., the one consisting of the polynomial predictors but without the mirror factor; $\chi^{2}=11.521, d D F=5, p<0.05$ ). Following Mirman (2017), we examined each individual interaction by dropping it from the full model and evaluated its unique effect by the reduction in the model fit.

We detected a significant effect of the MSR factor on the linear and quadratic term (see Table 2). This reflects the overall increase of pupil size in the recognisers compared to the nonrecognisers (the effect on linear term), that increases more quickly at the beginning of this time window (the effect on quadratic term), as we can observe in Figure 5. 
Early Effect. Through visual inspection of the data, we observed a difference in pupil reaction between the two MSR groups before our preregistered test period, at approximately the time when the agent begins to be occluded ( 5-6s). To explore this further, we conducted a functional t-test from $5 \mathrm{~s}$ to $6.5 \mathrm{~s}$ using permutation analysis at 0.05 significance level and 10000 permutations (see Figure 6), combining the data from both the HDfb and LDfb conditions as they are identical during this time period. We detected a statistically significant difference between the two groups from $5498 \mathrm{~ms}$ to $5820 \mathrm{~ms}$. Taking into account the pupil responsivity delay, this suggests that the mirror recognisers showed greater pupil dilation in anticipation of the agent becoming occluded than mirror non-recognisers ${ }^{1}$.

\section{Anticipatory Looking \& Inhibitory Control}

We examined whether, in a perspective-conflict scenario, a) infants' ability to look in anticipation to the correct window is modulated by their mirror self-recognition status, b) inhibitory control ability facilitates action prediction, and c) inhibitory control would only be a modulating factor once infants experience this conflict (i.e., only in mirror recognisers).

After pre-processing, infants in our sample watched a mean of $5.22 \pm 1.93 \mathrm{HDfb}$ and $5.08 \pm$ 1.84 LDfb trials. There was no significant difference in the number of trials included per condition $(t(98)=0.37, p=0.71)$. When split by MSR status, mirror non-recognisers had more trials included in both the HDfb condition $\left(M_{\text {recognisers }}=4.68 \pm 1.95, M_{\text {non-recognisers }}=5.76 \pm 1.79, t(48)=2.04, p=.05\right)$, and $\operatorname{LDfb}$ condition $\left(M_{\text {recognisers }}=4.44 \pm 1.47, M_{\text {non-recognisers }}=5.72 \pm 1.97, t(48)=2.60, p=.01\right)$.

Our first measure of belief-based action prediction was whether infants anticipated correctly (i.e., whether after the cue onset, the first box they fixated on was the side where the agent should have expected to find the ball given their false belief). Overall, infants did not reliably predict correctly, with first fixation scores in both conditions below chance $\left(M_{\mathrm{HDfb}}=0.36 \pm 0.26, \mathrm{t}(49)=\right.$ -

\footnotetext{
${ }^{1}$ As infants saw multiple trials, they could in principle anticipate the forthcoming disappearance of the agent at the same time on each trial.
} 
$3.70, \mathrm{p}<.001 ; M_{\mathrm{LDfb}}=0.41 \pm 0.23, \mathrm{t}(49)=-2.82, \mathrm{p}=.003$; both tests are one-tailed one-sample $\mathrm{t}-$ tests on first fixation scores below chance (.5)).

Our secondary measure of anticipatory looking was DLS score during the anticipation period. The mean DLS score in the HDfb condition was $-0.18 \pm 0.43$ and $-0.17 \pm 0.38$ in the LDfb condition. A score above 0.5 would have indicated longer looking to the correct AOI. One-sample t-tests on the DLS scores below chance (.5) indicated that infants looked longer at the incorrect $A O I$ than the correct AOI $(t(49)=-11.06, p<.001$ and $t(49)=-12.26, p<.001$, for the HDfb and LDfb conditions respectively).

Both recognisers and non-recognisers made anticipatory saccades in the same proportion of trials in both conditions (HDfb: $M_{\text {recognisers }}=0.84 \pm 0.21, M_{\text {non-recognisers }}=0.92 \pm 0.11, \mathrm{t}(36)=1.76, \mathrm{p}=$ .09 , Welch's test; LDfb: $M_{\text {recognisers }}=0.81 \pm 0.20, M_{\text {non-recognisers }}=0.88 \pm 0.15, \mathrm{t}(44)=1.36, \mathrm{p}=.18$, Welch's test).

In the absence of any evidence for correct anticipation, it is not informative to further examine differences in anticipatory looking between groups, or its relationship to inhibitory control abilities. However, we nonetheless include the preregistered analyses in the supplemental information.

\section{Latency}

\section{Anticipation phase}

Although infants did not make correct anticipatory saccades, we considered post hoc whether examining differences in latency to make anticipatory looks would allow us to better interpret the differences in dilation during the anticipation phase. Saccades during the anticipation window could reflect either only a reaction to exogenous cues (e.g., the lids on the box opening or the lights on the boxes that appear at the start of this window to prompt anticipation), or in addition, could reflect infants' genuine expectations concerning in which box they think the agent will search. While neither group provided any evidence that they correctly anticipated the agent's search, we reasoned that latency to make the initial saccade could provide information on whether 
anticipatory saccades were exogenously or endogenously mediated. Specifically, it is possible that a longer latency to generate a saccade to a box in the anticipatory window could reflect infants' attempt to manage the two conflicting representations. To examine latency, we computed the time from the box opening cue to the first saccade to one of the box AOIs. Independent samples t-tests (with unequal variances) revealed that mirror recognisers were indeed slower $(M=879.05 \pm$ 495.64ms) than non-recognisers $(M=652.97 \pm 254.00 \mathrm{~ms})$ in the HDfb condition $(t(35.79)=-2.03, p$ $=.05)$. Latency was not significantly different in the LDfb condition $\left(M_{\text {recognisers }}=810.13 \pm 453.00\right.$; $\left.M_{\text {non-recognisers }}=723.23 \pm 250.41 ; t(37.42)=-0.84, p=.41\right)$.

\section{Early effect}

We also considered posthoc whether examining differences in latency to making a saccade to the agent would allow us to better interpret the differences in pupil dilation during the earlier time window, just before the agent is occluded. We reasoned that if pupil dilation reflects anticipation of the agent being occluded, we may observe differences in how quickly infants saccade to the agent. Latency was computed as the time from the curtains beginning to close to the first saccade to the agent AOI in both the HDfb and LDfb conditions combined. An independent samples t-test revealed that mirror recognisers were faster $(M=540.71 \pm 270.58 \mathrm{~ms})$ than non-recognisers $(M=712.38 \pm 248.22 \mathrm{~ms})$ to saccade to the agent $(\mathrm{t}(48)=2.34, p=.02, d=.66)$.

\section{Discussion}

This study tested the hypothesis that the emergence of self-representation, between 18 and 24 months, creates a new challenge for young children when they are presented with a situation in which the self and other should hold conflicting perspectives (Southgate, 2020). Under this account, self-representation leads children to represent their own perspective as the self-perspective, and in doing so, this representation can compete with the representation of an event from the other's perspective. Thus, we preregistered the prediction that infants who had achieved self-awareness, as indexed by MSR, would experience greater conflict, as indexed by pupil dilation, while watching events in which conflict between self and other perspectives is high. We further predicted that this 
conflict may manifest in a reduced ability to make anticipatory saccades towards a location congruent with an agent's false belief in infants who evidenced MSR, but that this may be modulated by infants' capacity for inhibitory control.

Our functional data analysis, supported by growth curve analysis, indeed suggests that mirror recognisers perceived greater conflict during the late pre-registered time window (corresponding to the phase where they could expect the agent to reach), specifically in the high demand condition in which the conflict between self and other should be greatest. There were no differences in pupil dilation between recognisers and non-recognisers in the low demand condition. Within the high demand condition, mirror recognisers also took longer to saccade to a box during this anticipation phase. While latency was not a pre-registered variable, it could be interpreted as additional support for our interpretation of the pupil dilation data that it is the mirror recognisers who detect the perspective conflict, and this is reflected in their slower attempt at anticipation.

However, while we found the expected pupil dilation for recognisers in the high conflict condition, suggesting that it was only this group and this condition that the conflict between the two perspectives was perceived, we did not find support for our hypothesis that the non-recognisers would surpass the recognisers in correct action anticipation. To correctly anticipate, the infant would ostensibly need to inhibit their own conflicting perspective, particularly in the high-demand condition. In the current study, we found below-chance performance on both anticipatory looking measures, suggesting that both groups tended to look towards the last location of the ball. Considered in this context, the pupil data could suggest that 18-month-olds who have achieved selfawareness begin to perceive a conflict but are, as yet, unable to overcome this conflict. Alternatively, as recent studies have cast doubt on the reliability and validity of anticipatory looking as a measure of action prediction (Kampis et al., 2020), the absence of the predicted effect should be interpreted with caution.

In line with our pre-registered predictions, we have interpreted the greater pupil dilation in recognisers in the anticipatory window of the high-conflict condition as indicating that recognisers 
perceive greater conflict than non-recognisers, supporting our hypothesis that the emergence of self-representation may be an important factor in experiencing a conflict between the self and other perspectives. Similar to findings in adults (McCleery et al., 2011), the presence of a difference in pupil dilation only in this final window may imply that the perceived conflict between perspectives only emerges when the infant is cued to make a prediction about what the other person will do.

While we chose pupil dilation as a measure because of its relationship with conflict, it is important to note that we cannot be sure that pupil dilation itself reflects the process of perceiving or experiencing conflict. Pupil dilation has also been associated with both violation of expectation (Jackson \& Sirois, 2009) and effort (Kaldy \& Blaser, 2020) and it is not clear whether these processes are independent (Laeng et al., 2012). As we find this difference during the anticipatory time window, an alternative possibility is that it reflects the prediction process itself. While infants' tendency to make anticipatory saccades did not differ between recognisers and non-recognisers nor between high and low conflict trials, it is possible that the effort involved in anticipating was greater in recognisers on the high-demand condition, precisely because this is where recognisers perceived greatest conflict. However, even if this were the case, it would still provide support for our interpretation that the emergence of self-awareness is required for infants to experience a conflict when the self and other perspectives diverge.

Finally, a finding that emerged from our exploratory analyses is that recognisers also show significantly greater pupil dilation than non-recognisers at an early timepoint. During this window, infants see the curtains begin to occlude the agent from the scene, suggesting that recognisers and non-recognisers react to the disappearance of the agent differently. There are at least two possible ways of interpreting this finding. First, it may be that only the recognisers detect the significance of the disappearance of the agent, and it may be that this event is only worthy of attention if we understand the contrast with our own continued visual access. Alternatively, the difference between the recognisers and non-recognisers in pupil size during this time window could be driven by the non-recognisers' decreased interest in events that occur in the absence of other agents. According 
to the altercentric hypothesis, infants encode events better when they are co-witnessed with another agent, and thus events witnessed in the absence of others receive relatively less attention.

The data presented here provide initial support for the hypothesis that the emergence of self-representation presents a new challenge for infants' perspective tracking where they now need to manage two diverging perspectives in situations of perspective conflict. In doing so, our study may also provide evidence that infants' precocious perspective taking, prior to the onset of selfrepresentation, is possible precisely because they do not have to contend with conflicting perspectives. 


\section{References}

Amsterdam, B. (1972). Mirror self-image reactions before age two. Developmental Psychobiology: The Journal of the International Society for Developmental Psychobiology, 5(4), 297-305.

Asendorpf, J. B., \& Baudonnière, P.-M. (1993). Self-awareness and other-awareness: Mirror selfrecognition and synchronic imitation among unfamiliar peers. Developmental Psychology, 29(1), 88.

Baillargeon, R., Scott, R. M., \& He, Z. (2010). False-belief understanding in infants. Trends in Cognitive Sciences, 14(3), 110-118.

Birch, S. A., \& Bloom, P. (2007). The curse of knowledge in reasoning about false beliefs. Psychological Science, 18(5), 382-386.

Bulgarelli, C., Blasi, A., de Klerk, C. C., Richards, J. E., Hamilton, A., \& Southgate, V. (2019). Frontotemporoparietal connectivity and self-awareness in 18-month-olds: A resting state fNIRS study. Developmental Cognitive Neuroscience, 38, 100676.

Choi, Y.-j., Mou, Y., \& Luo, Y. (2018). How do 3-month-old infants attribute preferences to a human agent? Journal of experimental child psychology, 172, 96-106.

Community, B. O. (2018). Blender - a 3D modelling and rendering package. In Stichting Blender Foundation. http://www.blender.org

Devine, R. T., \& Hughes, C. (2014). Relations Between False Belief Understanding and Executive Function in Early Childhood: A Meta-Analysis. Child Development, 1777-1794. https://doi.org/10.1111/cdev.12237

Hartwright, C. E., Apperly, I. A., \& Hansen, P. C. (2015). The special case of self-perspective inhibition in mental, but not non-mental, representation. Neuropsychologia, 67, 183-192.

Hendry, A., Jones, E. J., \& Charman, T. (2016). Executive function in the first three years of life: Precursors, predictors and patterns. Developmental Review, 42, 1-33.

Hess, E. H., \& Polt, J. M. (1960). Pupil size as related to interest value of visual stimuli. Science, 132(3423), 349-350.

Holmboe, K., Larkman, C., de Klerk, C., Simpson, A., Bell, M. A., Patton, L., Christodoulou, C., \& Dvergsdal, H. (2021). The early childhood inhibitory touchscreen task: A new measure of response inhibition in toddlerhood and across the lifespan. PLoS One, 16(12), e0260695. https://doi.org/10.1371/journal.pone.0260695

Jackson, I., \& Sirois, S. (2009). Infant cognition: going full factorial with pupil dilation. Developmental Science, 12(4), 670-679.

Kahneman, D., \& Beatty, J. (1966). Pupil diameter and load on memory. Science, 154(3756), 15831585.

Kaldy, Z., \& Blaser, E. (2020). Putting effort into infant cognition. Current directions in psychological science, 29(2), 180-185.

Kampis, D., Karman, P., Csibra, G., Southgate, V., \& Hernik, M. (2020). A two-lab direct replication attempt of Southgate, Senju, \& Csibra (2007).

Kampis, D., Wiesmann, C. G., Koop, S., \& Southgate, V. (2021). Understanding the self in relation to others: Infants spontaneously map another's face to their own at 16 to 26 months. Developmental Science. https://doi.org/10.1111/desc.13197

Kret, M. E., \& Sjak-Shie, E. E. (2019). Preprocessing pupil size data: Guidelines and code. Behavior research methods, 51(3), 1336-1342.

Kuchinsky, S. E., Ahlstrom, J. B., Vaden Jr, K. I., Cute, S. L., Humes, L. E., Dubno, J. R., \& Eckert, M. A. (2013). Pupil size varies with word listening and response selection difficulty in older adults with hearing loss. Psychophysiology, 50(1), 23-34.

Laeng, B., Sirois, S., \& Gredebäck, G. (2012). Pupillometry:A Window to the Preconscious? Perspectives on Psychological Science, 7(1), 18-27. https://doi.org/10.1177/1745691611427305 
Leslie, A. M., Friedman, O., \& German, T. P. (2004). Core mechanisms in 'theory of mind'. Trends in Cognitive Sciences, 8(12), 528-533. https://doi.org/10.1016/j.tics.2004.10.001

Lewis, M., \& Ramsay, D. (2004). Development of self-recognition, personal pronoun use, and pretend play during the 2nd year. Child Development, 75(6), 1821-1831.

Luo, Y., \& Baillargeon, R. (2007). Do 12.5-month-old infants consider what objects others can see when interpreting their actions? Cognition, 105(3), 489-512.

Luo, Y., \& Johnson, S. C. (2009). Recognizing the role of perception in action at 6 months. Developmental Science, 12(1), 142-149.

McCleery, J. P., Surtees, A. D., Graham, K. A., Richards, J. E., \& Apperly, I. A. (2011). The neural and cognitive time course of theory of mind. J Neurosci, 31(36), 12849-12854. https://doi.org/10.1523/JNEUROSCI.1392-11.2011

McGarrigle, R., Dawes, P., Stewart, A. J., Kuchinsky, S. E., \& Munro, K. J. (2017). Pupillometry reveals changes in physiological arousal during a sustained listening task. Psychophysiology, 54(2), 193-203.

Mirman, D. (2017). Growth curve analysis and visualization using R. Chapman and Hall/CRC.

Müller, U., \& Kerns, K. (2015). The development of executive function. In Handbook of child psychology and developmental science: Cognitive processes, Vol. 2, 7th ed. (pp. 571-623). John Wiley \& Sons, Inc. https://doi.org/10.1002/9781118963418.childpsy214

Pätzold, W., \& Liszkowski, U. (2019). Pupillometry reveals communication-induced object expectations in 12-but not 8-month-old infants. Developmental Science, 22(6), e12832.

Ramsay, J. O., \& Silverman, B. W. (2002). Applied functional data analysis: methods and case studies (Vol. 77). Springer.

Rondeel, E., Van Steenbergen, H., Holland, R., \& van Knippenberg, A. (2015). A closer look at cognitive control: Differences in resource allocation during updating, inhibition and switching as revealed by pupillometry. Frontiers in human neuroscience, 9, 494.

Samson, D., Apperly, I. A., Kathirgamanathan, U., \& Humphreys, G. W. (2005). Seeing it my way: a case of a selective deficit in inhibiting self-perspective. Brain, 128(5), 1102-1111. https://doi.org/10.1093/brain/awh464

Schneider, D., Lam, R., Bayliss, A. P., \& Dux, P. E. (2012). Cognitive load disrupts implicit theory-ofmind processing. Psychol Sci, 23(8), 842-847. https://doi.org/10.1177/0956797612439070

Sirois, S., \& Brisson, J. (2014). Pupillometry. Wiley Interdisciplinary Reviews: Cognitive Science, 5(6), 679-692.

Southgate, V. (2020). Are infants altercentric? The other and the self in early social cognition. Psychological Review, 127(4), 505-523. https://doi.org/10.1037/rev0000182

Southgate, V., \& Vernetti, A. (2014). Belief-based action prediction in preverbal infants. Cognition, 130(1), 1-10. https://doi.org/10.1016/j.cognition.2013.08.008

Verschoor, S., Paulus, M., Spapé, M., Biro, S., \& Hommel, B. (2015). The developing cognitive substrate of sequential action control in 9-to 12-month-olds: Evidence for concurrent activation models. Cognition, 138, 64-78.

Wagner, A. E., Nagels, L., Toffanin, P., Opie, J. M., \& Başkent, D. (2019). Individual variations in effort: Assessing pupillometry for the hearing impaired. Trends in hearing, 23, 2331216519845596

Wang, L., \& Leslie, A. M. (2016). Is implicit theory of mind the 'Real Deal'? The own-belief/true-belief default in adults and young preschoolers. Mind \& Language, 31(2), 147-176.

Winn, M. B. (2016). Rapid release from listening effort resulting from semantic context, and effects of spectral degradation and cochlear implants. Trends in hearing, 20, 2331216516669723. 


\section{Acknowledgements}

This work was supported by a Consolidator Grant from the ERC (DEVOMIND 726114) to V.

Southgate. The authors thank Maria Bolding Eskildsen, Kathrine Habdank, Marie Louise Krogsgård Nielsen, and Kathrine Søndergaard Christensen for their assistance with testing, and Karla Holmboe and Henrik Dvergsdal for their assistance with implementing the ECITT.

\section{Author Contributions}

E. Yeung and V. Southgate developed the study concept. All authors contributed to the study design.

V. Manea and E. Yeung designed the stimuli. Data collection was performed by E. Yeung. D. Askitis and $\mathrm{E}$. Yeung performed the data analysis and interpretation under the supervision of V. Southgate. E. Yeung, D. Askitis, and V. Southgate wrote the manuscript. All authors approved the final version of the manuscript for submission. 


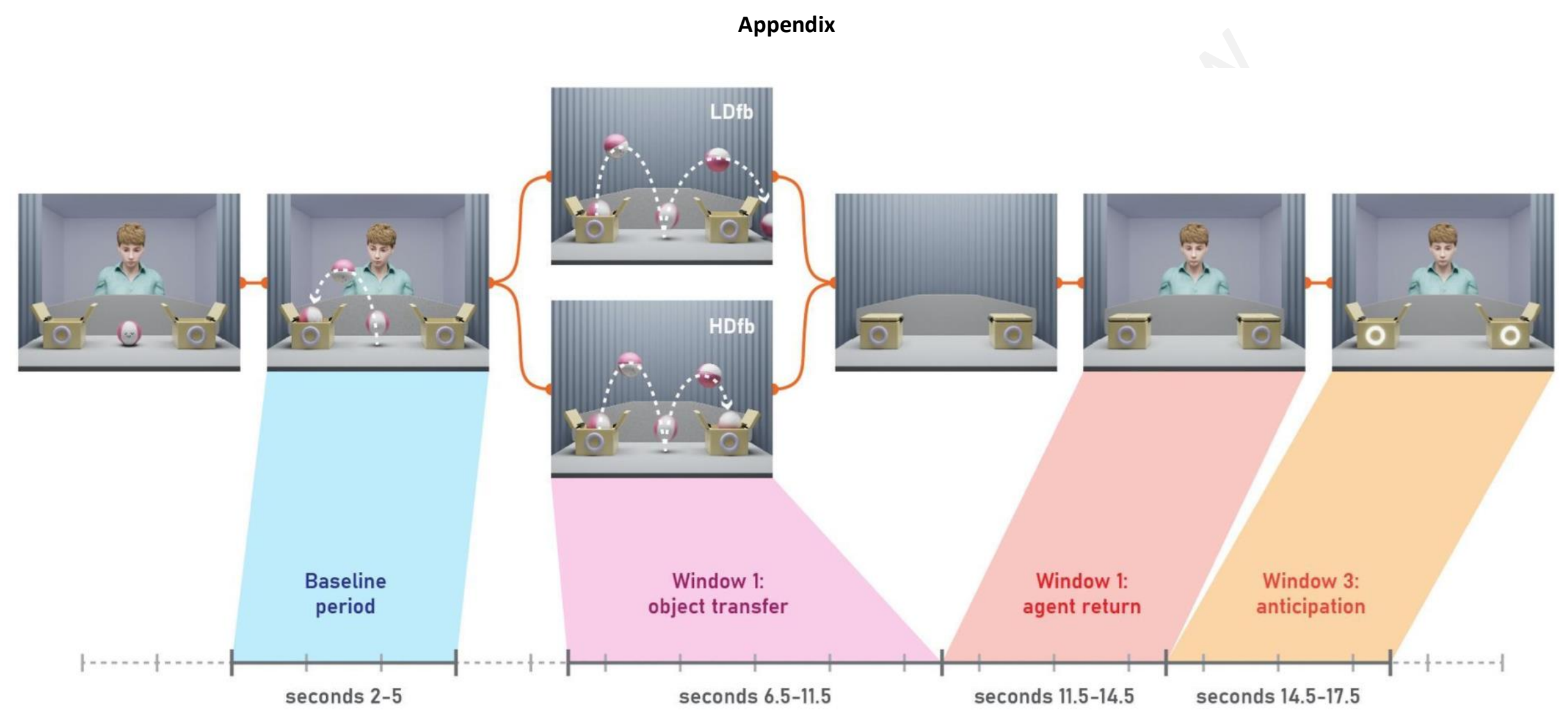

Figure 1. Time course and structure of the Non-Verbal Mentalizing Task. 
mirror - non-recognisers - recognisers

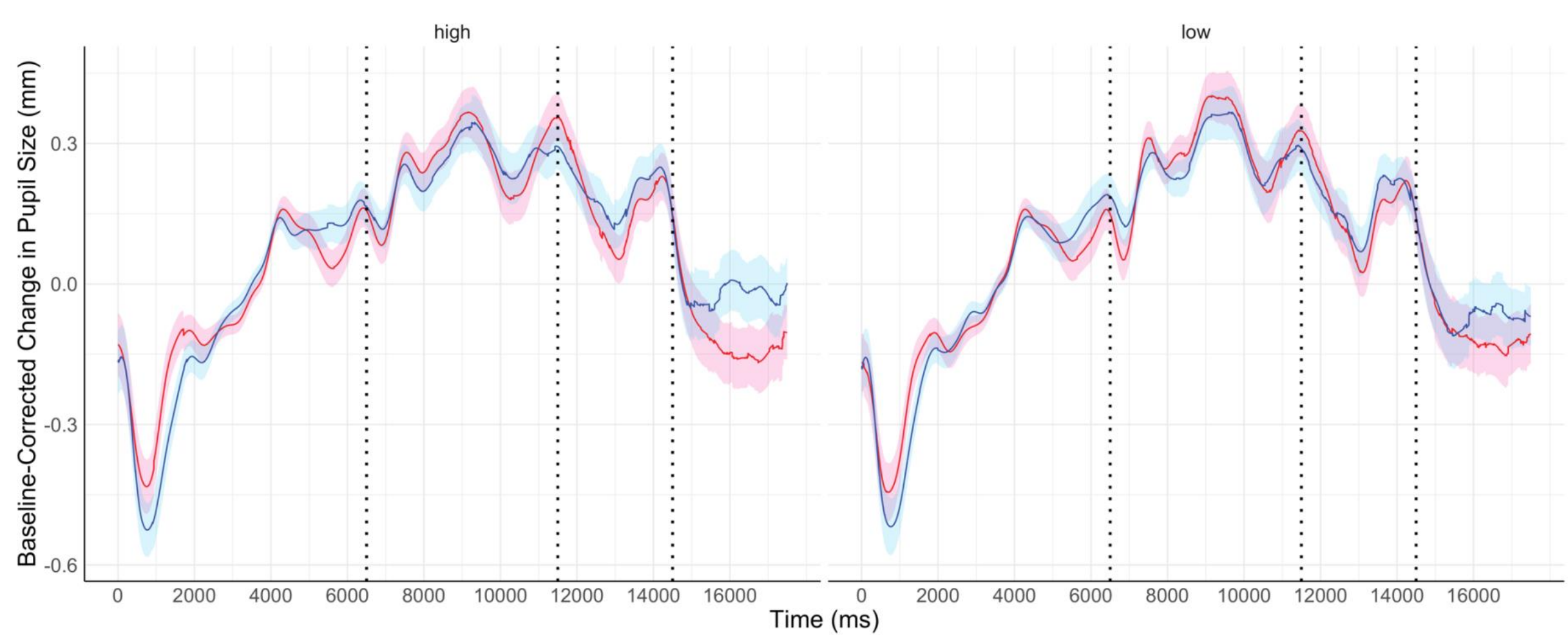

Figure 2. Baseline-Corrected Change in Pupil Size Across the duration of the Trial. The panel on the left shows the HDfb condition, the panel on the right shows the LDfb condition. Shaded regions show $95 \%$ confidence intervals. 


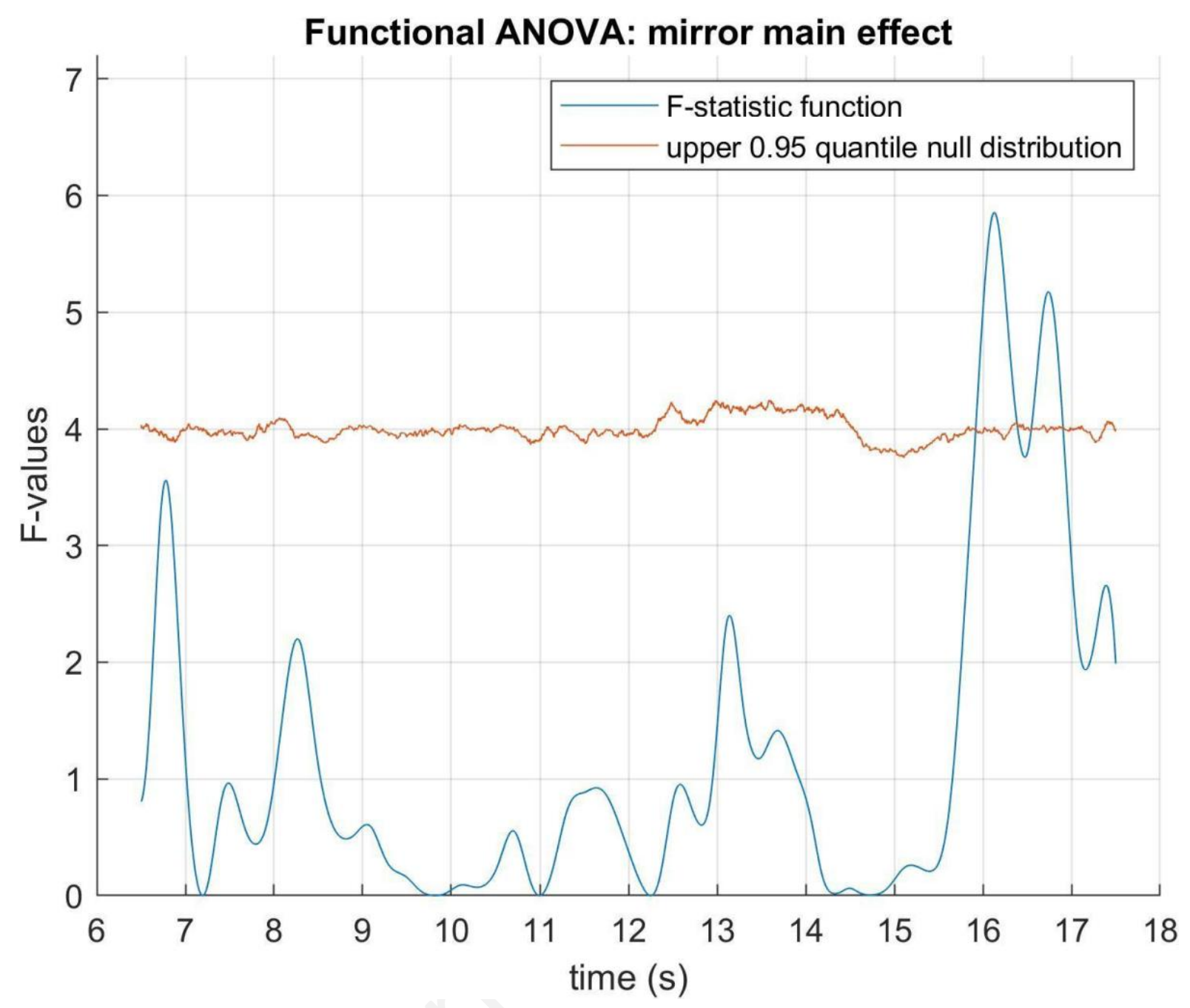

Figure 3a: Functional F-test (ANOVA) with MSR status as the between-subjects factor. Statistical significance is detected during the outcome phase, between 15918-16384 and 16544-16904 milliseconds. 


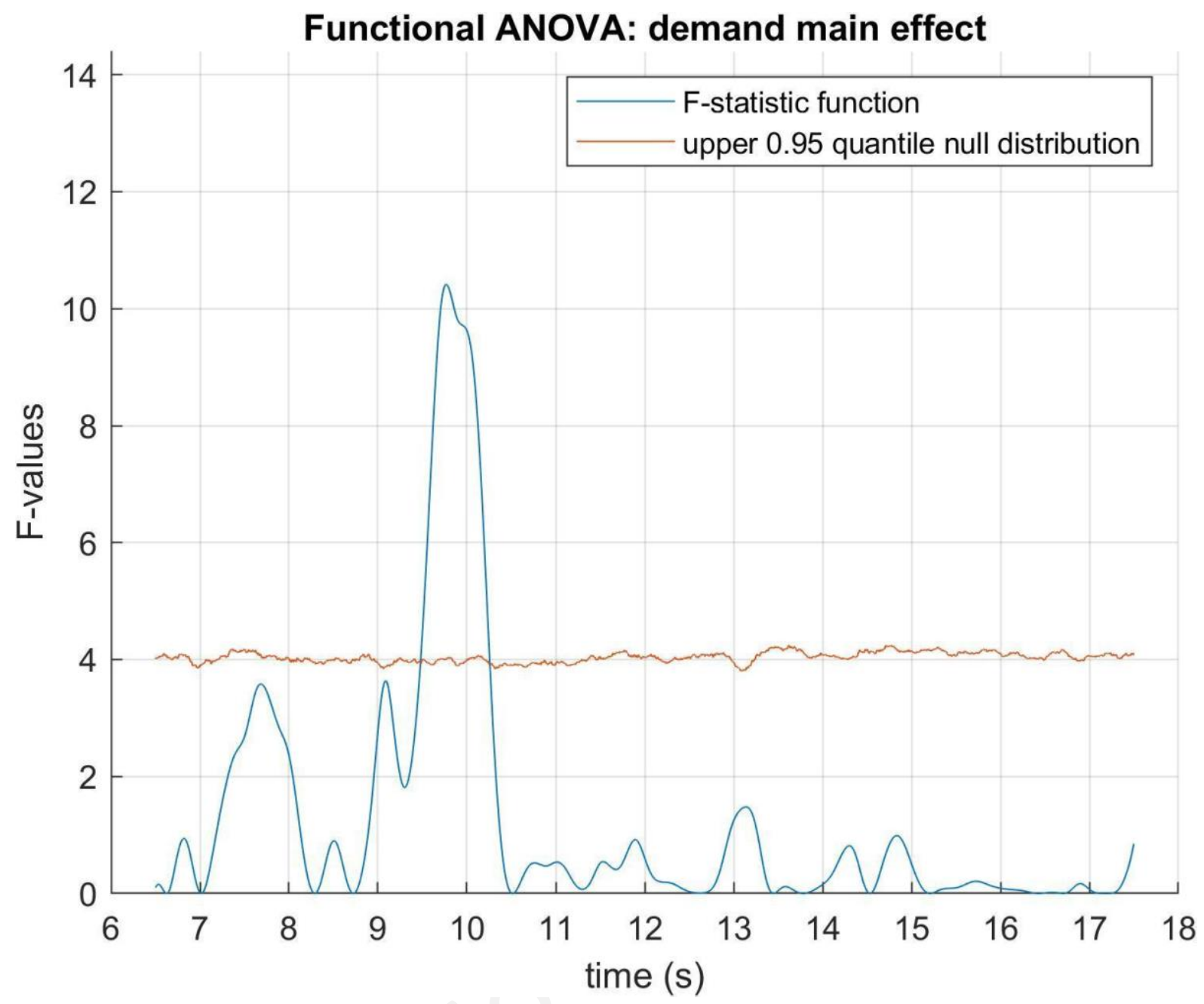

Figure 3b: Functional F-test (ANOVA) with condition (HDfb vs. LDfb) as the between-subjects factor. Statistical significance is detected only around the time period when the two conditions differ visually (i.e., the ball jumping into the second box or jumping out of the screen). 


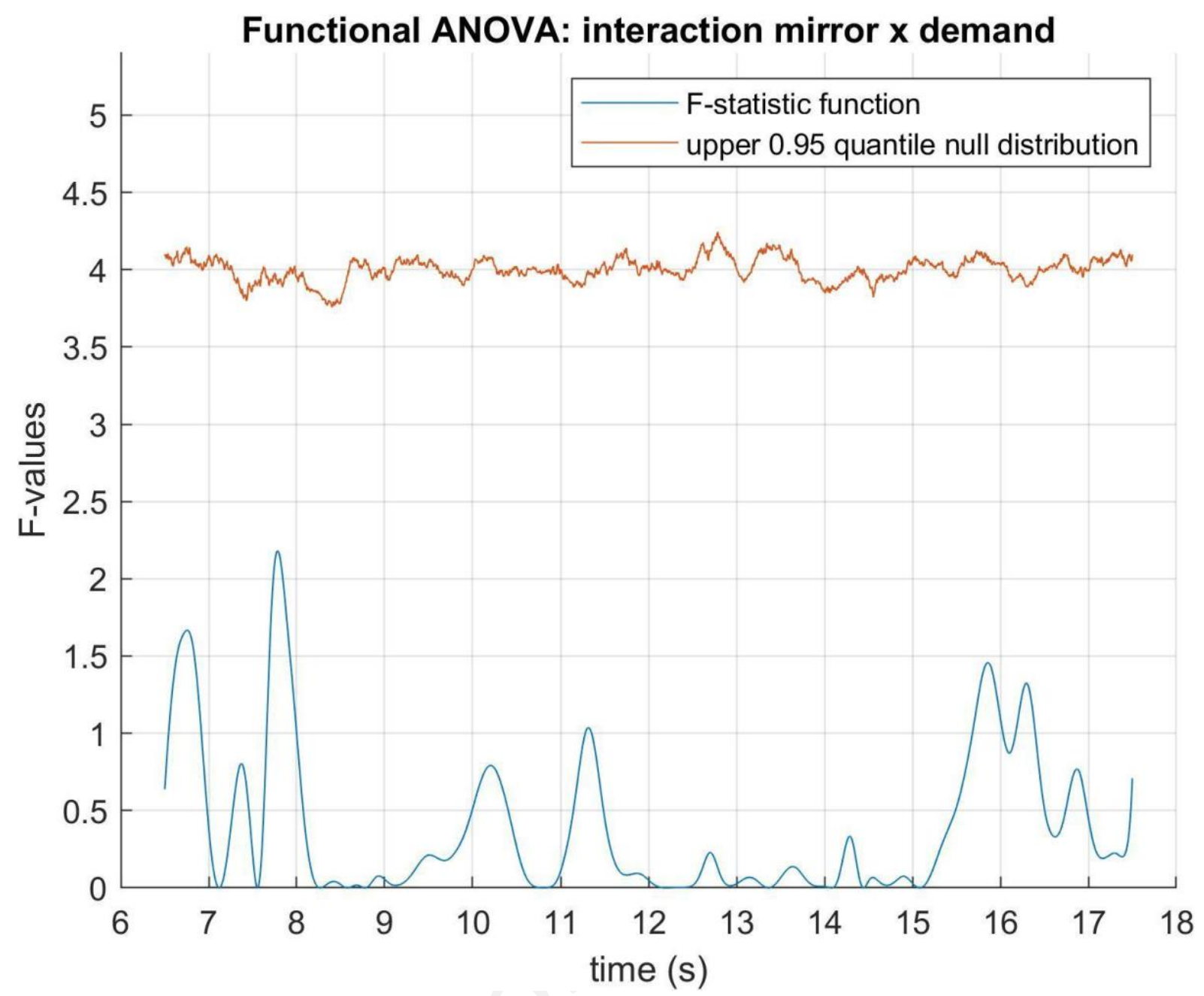

Figure 3c: Functional F-test (ANOVA) for the interaction between MSR status and demand condition. No statistical significance is detected. 


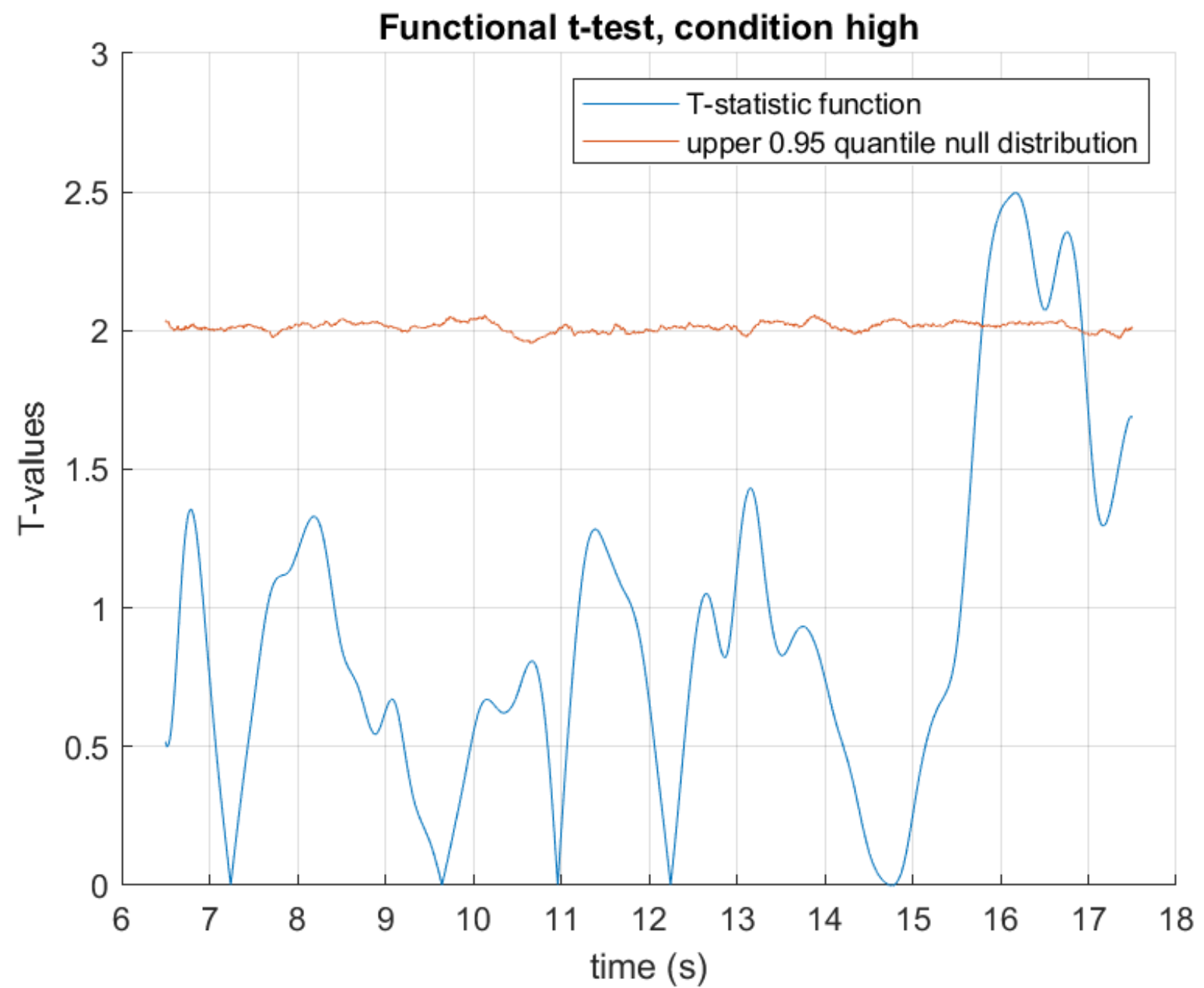

Figure 4a: Functional t-test for mirror recognisers vs non-recognisers within the high demand condition. Statistically significant differences are observed during the outcome phase, from 15790 to 16990 milliseconds. 
4

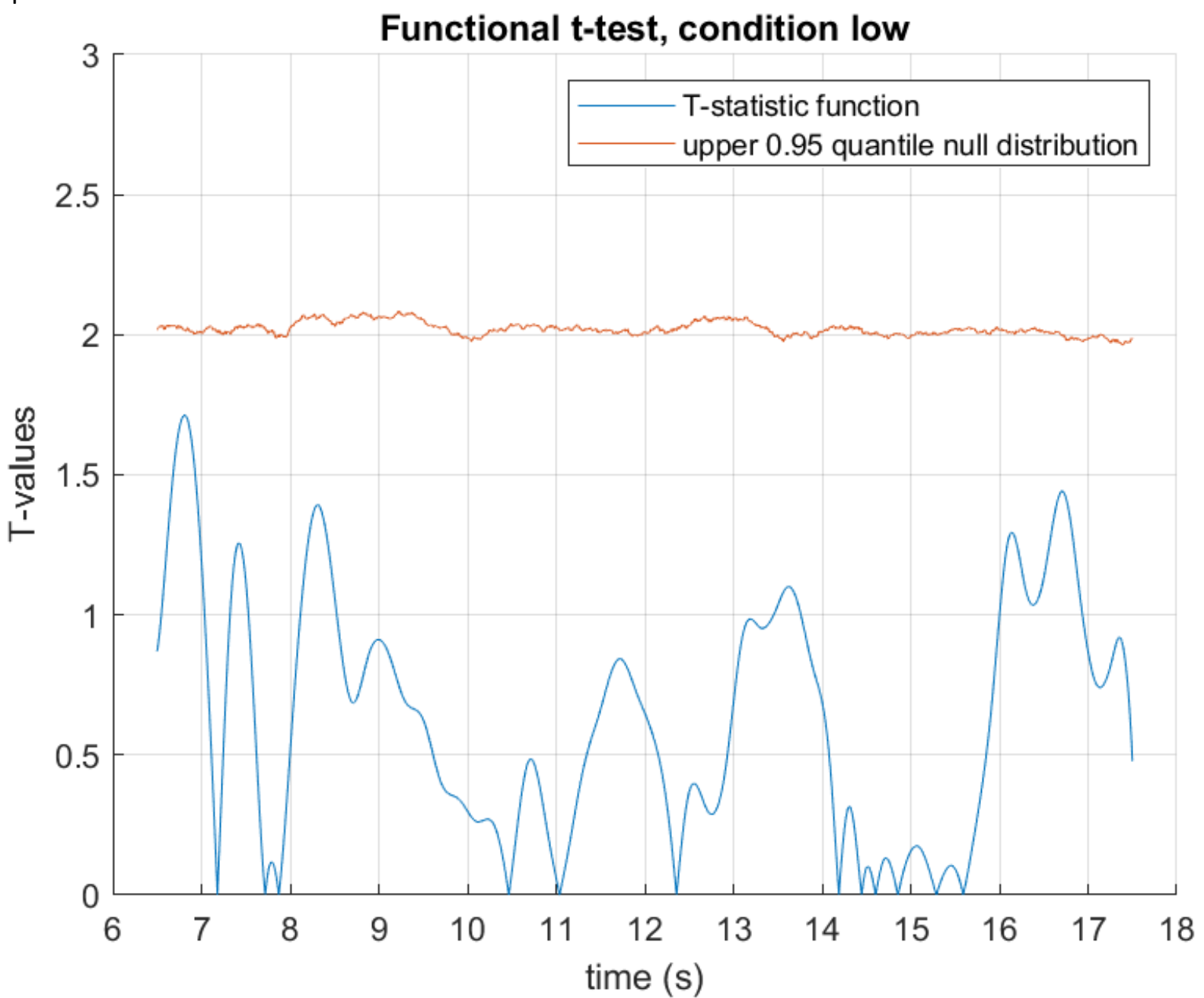

Figure 4b: Functional T-test for mirror recognisers vs. non-recognisers within the low demand condition. No statistically significant differences were observed across the test phase. 


\begin{tabular}{lrrrr}
\hline \multicolumn{1}{r}{ Term } & Estimate & SE & $t$ & $p$ \\
\hline (Intercept) & -0.05938 & 0.02913 & -2.03846 & 0.041504 \\
op1 & -0.24622 & 0.078655 & -3.13042 & 0.001745 \\
op2 & 0.24644 & 0.058157 & 4.237516 & 0.000226 \\
op3 & -0.14852 & 0.041083 & -3.61516 & 0.000300 \\
op4 & 0.125062 & 0.034271 & 3.649185 & 0.000263 \\
mirror & 0.046273 & 0.02913 & 1.588465 & 0.112180 \\
mirror:op1 & 0.158428 & 0.078655 & 2.014229 & $0.043985 *$ \\
mirror:op2 & -0.12716 & 0.058157 & -2.18647 & $0.028781^{*}$ \\
mirror:op3 & -0.05075 & 0.041083 & -1.23531 & 0.21672 \\
mirror:op4 & 0.047509 & 0.034271 & 1.38627 & 0.16566 \\
\hline
\end{tabular}

Table 1. The full model within the high demand condition, with pupil mirror* (op1 + op $2+$ op $3+$ op4) $+(o p 1+o p 2+o p 3+o p 4 \mid s u b j)$. For the calculation of the p-values the z-distribution is used as an approximation to the t-distribution (see Mirman, 2017). We see the effect terms of mirror group on the linear and quadratic term are significant.

\begin{tabular}{lcc}
\hline interaction term dropped & $\chi^{2}$ & $p$ \\
\hline linear & 3.898 & $0.0483^{*}$ \\
quadratic & 4.561 & $0.0327^{*}$ \\
cubic & 1.503 & 0.2203 \\
quartic & 1.885 & 0.1698 \\
\hline
\end{tabular}

Table 2: The effect of dropping each single interaction of a polynomial term with mirror group to model fitness. Because we drop only one term each time, the degree of freedom is one. We see that dropping the first two terms results in worse fitting models, which let us conclude that these terms are significant. 


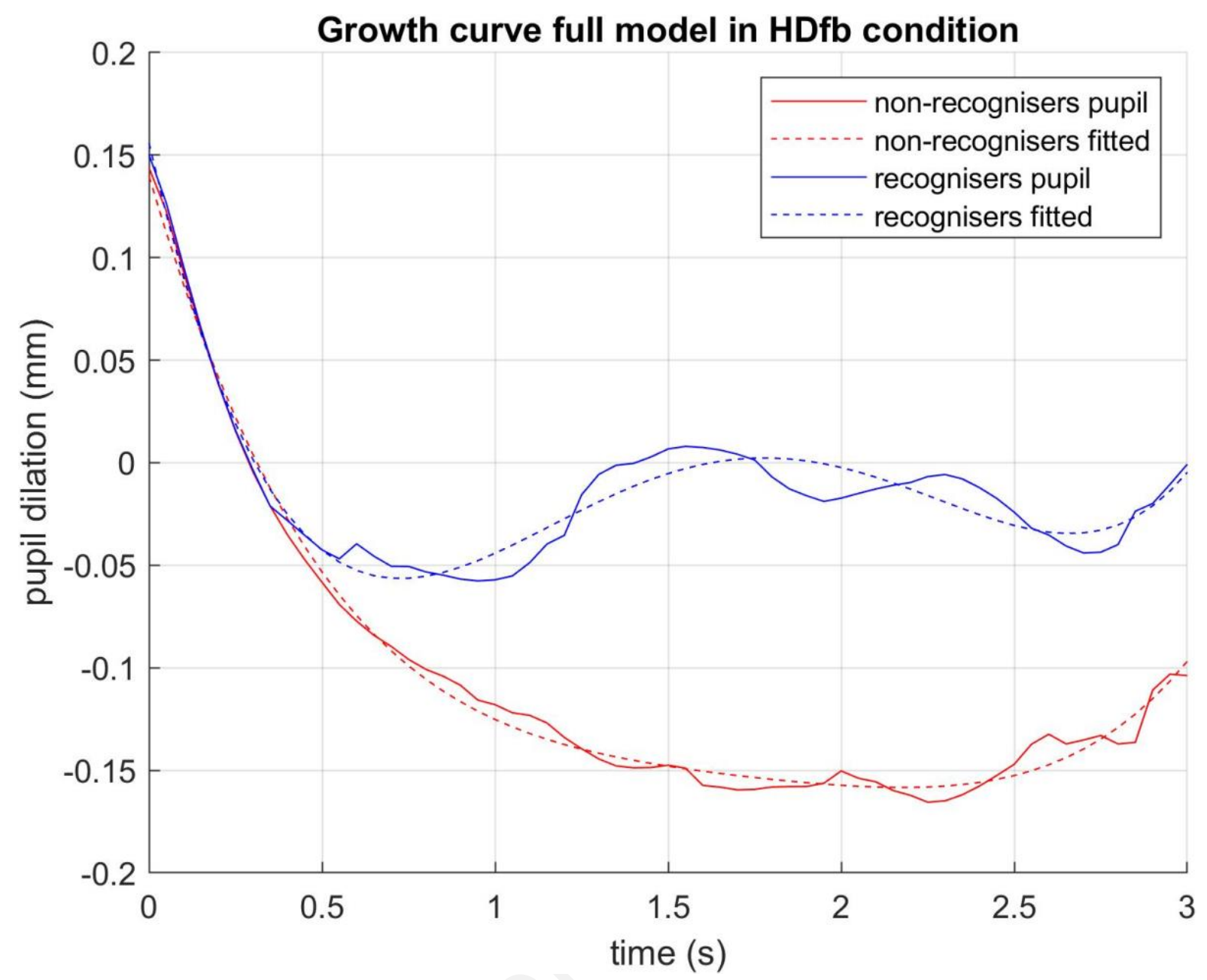

Figure 5. Plotted fit of the full growth curve model, between mirror recognition groups and within the high demand condition. 


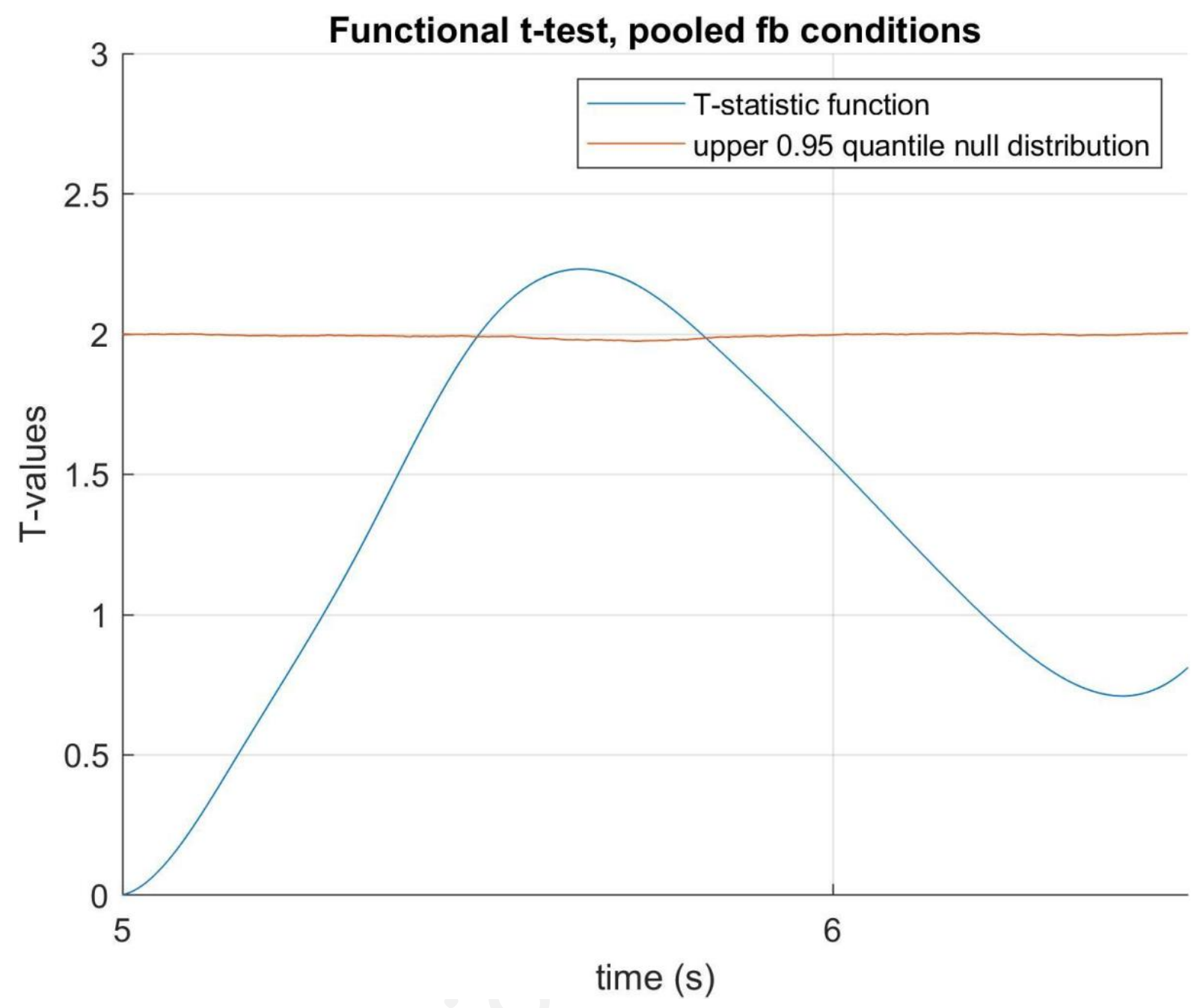

Figure 6: Functional T-test for mirror self-recognisers vs non-recognisers in both conditions in the early period around agent occlusion. We detect a significant difference from $5498 \mathrm{~ms}$ to $5820 \mathrm{~ms}$. 